\title{
TRIDIAGONAL MATRIX REPRESENTATIONS OF CYCLIC SELF-ADJOINT OPERATORS. II
}

\section{J. DOMBROWSKI}

\begin{abstract}
A bounded cyclic self-adjoint operator $C$ defined on a separable Hilbert space $H$ can be represented as a tridiagonal matrix with respect to the basis generated by the cyclic vector. An operator $J$ can then be defined so that $C J-J C=-2 i K$ where $K$ also has tridiagonal form. If the subdiagonal elements of $C$ converge to a non-zero limit and if $K$ is of trace class then $C$ must have an absolutely continuous part.
\end{abstract}

1. Introduction. A bounded cyclic self-adjoint operator $C$ defined on a separable Hilbert space $H$ has a tridiagonal matrix representation with respect to the basis generated by the cyclic vector. The spectral properties of $C$ are studied in [1] under the assumption that the main diagonal elements in the tridiagonal representation are zeros. In this case $C$ is the real part of a weighted shift operator, and if $J$ is the corresponding imaginary part then $C J-J C=2 i K$ where $K$ is diagonal. It is shown in [1] that $C$ has an absolutely continuous part if $K$ is of trace class.

The purpose of this paper is to extend the above result to tridiagonal matrices with non-zero diagonal. This extension is significant for the study of systems of orthogonal polynomials which satisfy a three term recursion formula. The coefficients in the recursion formula correspond to a unique tridiagonal matrix whose spectral measure is the measure of orthogonality for the system of polynomials. If the measure is symmetric about the origin (as in the case of the normalized Legendre polynomials) then the diagonal entries of the corresponding tridiagonal matrix will be zero. See [1] for further background.

Suppose now that $C$ is a bounded cyclic self-adjoint operator with the following tridiagonal matrix representation with respect to the basis $\left\{\phi_{n}\right\}$ :

$$
C=\left[\begin{array}{ccccc}
b_{1} & a_{1} & 0 & 0 & \cdots \\
a_{1} & b_{2} & a_{2} & 0 & \cdots \\
0 & a_{2} & b_{3} & a_{3} & \cdots \\
\vdots & \vdots & \vdots & \vdots &
\end{array}\right], \quad a_{n}>0 .
$$

Then $\phi_{n}=P_{n}(C) \phi_{1}$ with $P_{1}(\lambda)=1, P_{2}(\lambda)=\left(\lambda-b_{1}\right) / a_{1}$ and for $n>2$,

$$
P_{n}(\lambda)=\frac{\left(\lambda-b_{n-1}\right) P_{n-1}(\lambda)-a_{n-2} P_{n-2}(\lambda)}{a_{n-1}} \text {. }
$$


It follows that $C$ is the real part of the operator $T$ defined by $T \phi_{n}=b_{n} \phi_{n}$ $+2 a_{n} \phi_{n+1}$. If $J=(1 / 2 i)\left(T+T^{*}\right)$ then $C J-J C=-2 i K$ where $K=$ $\left[k_{i j}\right]$ has tridiagonal form with

$$
\begin{aligned}
& k_{i i}=-a_{i-1}^{2}+a_{i}^{2} \quad\left(a_{0}=0\right), \\
& k_{i, i+1}=k_{i+1, i}=\frac{1}{2} a_{i}\left(b_{i}-b_{i+1}\right) .
\end{aligned}
$$

The aim now is to analyze the spectrum of $C$ if $\sum\left|k_{i i}\right|<\infty$ and $\sum\left|k_{i, i+1}\right|$ $<\infty$. Note that in this case $\lim a_{n}$ and $\lim b_{n}$ exist. If $\lim a_{n}=\alpha(\alpha \neq 0)$ and $\lim b_{n}=0$ then the spectrum of $C$ at least contains the interval $(-2 \alpha, 2 \alpha)$. Note also that the diagonals of $K$ are absolutely summable whenever $K \geq 0$ so that $T$ is hyponormal. For if $K=\left[k_{i j}\right]$ is positive then $\left\{a_{n}^{2}\right\}$ is an increasing sequence and $\left|k_{i j}\right|^{2} \leq\left|k_{i i}\right|\left|k_{j j}\right|$. It follows that

$$
\frac{1}{2} \sum\left|a_{i}\right|\left|b_{i+1}-b_{i}\right| \leq\left(\sum\left|-a_{i-1}^{2}+a_{i}^{2}\right|\right)^{1 / 2}\left(\sum\left|-a_{i}^{2}+a_{i+1}^{2}\right|\right)^{1 / 2} .
$$

Furthermore, the following is true:

THEOREM. The operator $K$ in (1.2) is of trace class if and only if $\sum\left|k_{i i}\right|<\infty$ and $\sum\left|k_{i, i+1}\right|<\infty$.

Proof. Assume that the diagonals of $K$ are absolutely summable. It follows from Jensen's Inequality that

$$
\sum\left\langle\left(K^{*} K\right)^{1 / 2} \phi_{n}, \phi_{n}\right\rangle \leq \sum\left\langle K^{*} K \phi_{n}, \phi_{n}\right\rangle^{1 / 2}
$$

and a direct computation shows that if $K^{*} K=\left[p_{i j}\right]$ then

$$
\begin{aligned}
p_{11}=a_{1}^{4} & +\frac{1}{4}\left(-a_{1} b_{1}+a_{1} b_{2}\right)^{2}, \\
p_{i i}= & \frac{1}{4}\left(-a_{i-1} b_{i-1}+a_{i-1} b_{i}\right)^{2}+\left(-a_{i-1}^{2}+a_{i}^{2}\right)^{2} \\
& +\frac{1}{4}\left(-a_{i} b_{i}+a_{i} b_{i+1}\right)^{2} \text { for } i>1 .
\end{aligned}
$$

Since

$$
\begin{aligned}
\sum p_{i i}^{1 / 2} \leq & \sqrt{2}\left(a_{1}^{2}+\frac{1}{2}\left|-a_{1} b_{1}+a_{1} b_{2}\right|\right) \\
& +\sqrt{3} \sum\left(\frac{1}{2}\left|-a_{i-1} b_{i-1}+a_{i-1} b_{i}\right|+\left|-a_{i-1}^{2}+a_{i}^{2}\right|\right. \\
& \left.+\frac{1}{2}\left|-a_{i} b_{i}+a_{i} b_{i+1}\right|\right),
\end{aligned}
$$

$K$ is of trace class.

Suppose $K$ is of trace class. Write $K=D+W$ where $D$ is diagonal and $W$ is the real part of a weighted shift operator. Since $\Sigma\left|\left\langle K \phi_{n}, \phi_{n}\right\rangle\right|<$ $\infty, D$ (and hence $W$ ) must be of trace class. Furthermore, $W$ with weight 
sequence $\left\{w_{i}\right\}$ is unitarily equivalent to $W^{\prime}$ with weight sequence $\left\{\left|w_{i}\right|\right\}$. If $V$ denotes the unilateral shift operator then $W^{\prime}\left(V+V^{*}\right)$ with diagonal elements $\left|w_{1}\right|,\left|w_{1}\right|+\left|w_{2}\right|,\left|w_{2}\right|+\left|w_{3}\right|, \ldots$, is of trace class. Hence the diagonals of $K$ are absolutely summable.

2. The absolutely continuous part of $\mathrm{C}$. If $C=\int \lambda d E_{\lambda}$ let $H_{a}(C)$ denote the set of $x$ in $H$ for which $\left\|E_{\lambda} x\right\|^{2}$ is an absolutely continuous function of $\lambda$. The subspace $H_{a}(C)$ reduces $C$ and the restriction of $C$ to $H_{a}(C)$ is called the absolutely continuous part of $C$. If $C$ is represented as a multiplication operator on $L^{2}(\mu)$ these ideas are related to the decomposition of the spectral measure $\mu$.

The following lemma is needed for the proof of the main result.

LEMMA. If

$$
\begin{aligned}
S_{N+1}(\lambda)=a_{1}^{2} P_{1}^{2}(\lambda)+\sum_{n=2}^{N+1}[ & \left.-a_{n-1}^{2}+a_{n}^{2}\right) P_{n}^{2}(\lambda) \\
& \left.+a_{n-1}\left(-b_{n-1}+b_{n}\right) P_{n-1}(\lambda) P_{n}(\lambda)\right]
\end{aligned}
$$

then

$$
S_{N+1}(\lambda)=a_{N}^{2} p_{N}^{2}(\lambda)-a_{N}\left(\lambda-b_{N+1}\right) P_{N}(\lambda) P_{N+1}(\lambda)+a_{N+1}^{2} P_{N+1}^{2}(\lambda) .
$$

Proof. Note that

$$
\begin{aligned}
S_{2}(\lambda)= & a_{1}^{2} P_{1}^{2}(\lambda)+a_{1}\left(-b_{1}+b_{2}\right) P_{1}(\lambda) P_{2}(\lambda) \\
& -a_{1}\left(\lambda-b_{1}\right) P_{2}(\lambda) P_{1}(\lambda)+a_{2}^{2} P_{2}^{2}(\lambda) \\
= & a_{1}^{2} P_{1}^{2}(\lambda)+a_{1}\left(b_{2}-\lambda\right) P_{1}(\lambda) P_{2}(\lambda)+a_{2}^{2} P_{2}^{2}(\lambda) .
\end{aligned}
$$

Assume that the result has been verified for $N=1, \ldots, k$ and recall that $a_{k} P_{k+1}(\lambda)=\left(\lambda-b_{k}\right) P_{k}(\lambda)-a_{k-1} P_{k-1}(\lambda)$. Then

$$
\begin{aligned}
S_{k+1}(\lambda)= & a_{k-1}^{2} P_{k-1}^{2}(\lambda)-a_{k-1}\left(\lambda-b_{k}\right) P_{k-1}(\lambda) P_{k}(\lambda) \\
& +a_{k}^{2} P_{k}^{2}(\lambda)-\left[\left(\lambda-b_{k}\right) P_{k}(\lambda)-a_{k-1} P_{k-1}(\lambda)\right]^{2} \\
& +a_{k+1}^{2} P_{k+1}^{2}(\lambda)+\left(-a_{k} b_{k}+a_{k} b_{k+1}\right) P_{k}(\lambda) P_{k+1}(\lambda) \\
= & a_{k}^{2} P_{k}^{2}(\lambda)-\left(\lambda-b_{k}\right) P_{k}(\lambda)\left[\left(\lambda-b_{k}\right) P_{k}(\lambda)-a_{k-1} P_{k-1}(\lambda)\right] \\
& +a_{k+1}^{2} P_{k+1}^{2}(\lambda)+\left(-a_{k} b_{k}+a_{k} b_{k+1}\right) P_{k}(\lambda) P_{k+1}(\lambda) \\
= & a_{k}^{2} P_{k}^{2}(\lambda)-a_{k}\left(\lambda-b_{k+1}\right) P_{k}(\lambda) P_{k+1}(\lambda)+a_{k+1}^{2} P_{k+1}^{2}(\lambda)
\end{aligned}
$$

THEOREM. Let $\lim a_{n}=1 / 2$ and $\lim b_{n}=0$. If $\sum\left|-a_{n-1}^{2}+a_{n}^{2}\right|<\infty$ and $\sum\left|a_{n-1}\left(-b_{n-1}+b_{n}\right)\right|<\infty$ then the spectral measure of $C$ is absolutely continuous on $(-1,1)$. 
Proof. The plan is to first consider an operator obtained from $C$ by replacing a finite number of constants in such a way as to simplify the structure of $K$ and make use of the absolute summability of the diagonals. Given $k>1$ choose $R(k)$ such that

$$
\sum_{R+1}^{\infty}\left|-a_{n-1}^{2}+a_{n}^{2}\right| \leq \frac{1}{32 k}, \quad \sum_{R+1}^{\infty}\left|a_{n-1}\left(-b_{n-1}+b_{n}\right)\right|<\frac{1}{32 k}
$$

and such that if $n>R$ then $\left|4 a_{n}^{2}-1\right|<1 / 16 k,\left|b_{n}\right|<1 / 16 k$. Obtain $C_{k}$ from $C$ by replacing the constants $b_{1}, \ldots, b_{R}$ by 0 and the constants $a_{1}, \ldots, a_{R}$ by $1 / 2$. It will now be shown that the spectral measure of $C_{k}$ is absolutely continuous on $(-1+1 / k, 1-1 / k)$. Since $C$ is a trace class perturbation of $C_{k}$ the same result will then hold for $C$.

Let $\Delta$ be a subinterval of $(-1+1 / k, 1-1 / k)$ and for $C_{k}=\int \lambda d E_{\lambda}$ let $\mu(\Delta)=\left\|E(\Delta) \phi_{1}\right\|^{2}$. With $J$ and $K$ defined as before,

$$
\begin{aligned}
& \left\langle K E(\Delta) \phi_{1}, E(\Delta) \phi_{1}\right\rangle \\
& =a_{1}^{2}\left|\left\langle E(\Delta) \phi_{1}, \phi_{1}\right\rangle\right|^{2} \\
& +\sum_{n=2}^{\infty}\left[a_{n-1}\left(-b_{n-1}+b_{n}\right)\left\langle E(\Delta) \phi_{1}, \phi_{n-1}\right\rangle\left\langle E(\Delta) \phi_{1}, \phi_{n}\right\rangle\right. \\
& \left.+\left(-a_{n-1}^{2}+a_{n}^{2}\right)\left|\left\langle E(\Delta) \phi_{1}, \phi_{n}\right\rangle\right|^{2}\right] \\
& =a_{1}^{2}\left|\int_{\Delta} P_{1} d \mu\right|^{2}+\sum_{n=2}^{\infty}\left[a_{n-1}\left(-b_{n-1}+b_{n}\right) \int_{\Delta} P_{n-1} d \mu \int_{\Delta} P_{n} d \mu\right. \\
& \left.+\left(a_{n-1}^{2}+a_{n}^{2}\right)\left|\int_{\Delta} P_{n} d \mu\right|^{2}\right] .
\end{aligned}
$$

Several cases need to be considered.

Case I. Suppose $\int_{\Delta} P_{n}^{2} d \mu \leq \mu(\Delta)=\int_{\Delta} P_{1}^{2} d \mu$ for each $n$. Then

$$
\begin{aligned}
& \left\langle K E(\Delta) \phi_{1}, E(\Delta) \phi_{1}\right\rangle \\
& \geq a_{1}^{2}|\mu(\Delta)|^{2}-\mu(\Delta) \sum_{n=M+1}^{\infty}\left[\left|a_{n-1}\left(-b_{n-1}+b_{n}\right)\right|\left(\int P_{n-1}^{2} d \mu\right)^{1 / 2}\right. \\
& \left.\times\left(\int P_{n}^{2} d \mu\right)^{1 / 2}+\left|-a_{n-1}^{2}+a_{n}^{2}\right| \int P_{n}^{2} d \mu\right] \\
& \geq|\mu(\Delta)|^{2}\left(\frac{1}{4}-\frac{1}{16 k}\right)
\end{aligned}
$$


Case II. Suppose $\int_{\Delta} P_{n}^{2} d \mu \leq \mu(\Delta)=\int_{\Delta} P_{1}^{2} d \mu$ except for a finite number of $n$. Then there exists $N$ such that $\int_{\Delta} P_{N}^{2} d \mu=\max \int_{\Delta} P_{n}^{2} d \mu$. Adding and substracting

$$
\mu(\Delta) \sum_{n=2}^{N} \int\left[a_{n-1}\left(-b_{n-1}+b_{n}\right) P_{n-1} P_{n}+\left(-a_{n-1}^{2}+a_{n}^{2}\right) P_{n}^{2}\right] d \mu
$$

to the expansion of $\left\langle K E(\Delta) \phi_{1}, E(\Delta) \phi_{1}\right\rangle$ in (2.1) and using the expression for $S_{N}$ given in the lemma it follows that

$$
\begin{aligned}
& \left\langle K E(\Delta) \phi_{1}, E(\Delta) \phi_{1}\right\rangle \\
& =\mu(\Delta)\left\{\int\left[a_{N-1} P_{N-1}(\lambda)-\frac{1}{2}\left(\lambda-b_{N}\right) P_{N}(\lambda)\right]^{2} d \mu\right. \\
& \left.\quad+\int\left[a_{N}^{2}-\frac{1}{4}\left(\lambda-b_{N}\right)^{2} P_{N}^{2}(\lambda)\right] d \mu\right\} \\
& \quad-\mu(\Delta) \sum_{n=2}^{N} \int\left[a_{n-1}\left(-b_{n-1}+b_{n}\right) P_{n-1} P_{n}+\left(-a_{n-1}^{2}+a_{n}^{2}\right) P_{n}^{2}\right] d \mu \\
& \quad+\sum_{n=2}\left[a_{n-1}\left(-b_{n-1}+b_{n}\right) \int_{\Delta} P_{n-1} d \mu \int_{\Delta} P_{n} d \mu\right. \\
& \left.\quad+\left(-a_{n-1}^{2}+a_{n}^{2}\right)\left|\int P_{n} d \mu\right|^{2}\right] \\
& \geq \frac{1}{4} \mu(\Delta) \int\left[4 a_{N}^{2}-\left(\lambda-b_{N}\right)^{2}\right] P_{N}^{2}(\lambda) d \mu-\frac{1}{8 k} \mu(\Delta) \int_{\Delta} P_{N}^{2} d \mu \\
& \geq|\mu(\Delta)|^{2} \frac{3}{32 k} .
\end{aligned}
$$

Case III. Suppose there exists a subsequence $\left\{P_{n j}\right\}$ such that $\int_{\Delta} P_{n j}^{2} d \mu$ $\geq \mu(\Delta)$. Choose $L$ sufficiently large such that

$$
\begin{aligned}
& \sum_{L+1}^{\infty}\left[a_{n-1}\left(-b_{n-1}+b_{n}\right) \int_{\Delta} P_{n-1} d \mu \int_{\Delta} P_{n} d \mu+\left(-a_{n-1}^{2}+a_{n}^{2}\right)\left|\int P_{n} d \mu\right|^{2}\right] \\
& \quad \leq \mu(\Delta) \sum_{L+1}^{\infty}\left[\left|a_{n-1}\left(-b_{n-1}+b_{n}\right)\right|+\left|-a_{n-1}^{2}+a_{n}^{2}\right|\right] \leq \frac{1}{32 k}|\mu(\Delta)|^{2}
\end{aligned}
$$

Choose $M>L$ such that $\int_{\Delta} P_{M}^{2} d \mu \geq \mu(\Delta)$. Then there exists $N$ such that $\int_{\Delta} P_{N}^{2} d \mu=\max _{1 \leq n \leq M} \int_{\Delta} P_{n}^{2} d \mu$. Using the same approach as in Case II it 
can be shown that

$$
\begin{aligned}
& \left\langle K E(\Delta) \phi_{1}, E(\Delta) \phi_{1}\right\rangle \\
& \geq \mu(\Delta) \int_{\Delta}\left[a_{N}^{2}-\frac{1}{4}\left(\lambda-b_{N}\right)^{2}\right] P_{N}^{2}(\lambda) d \mu \\
& -\mu(\Delta) \sum_{n=2}^{N} \int\left[a_{n-1}\left(-b_{n-1}+b_{n}\right) P_{n-1} P_{n} d \mu+\left(-a_{n-1}^{2}+a_{n}^{2}\right) P_{n}^{2}\right] d \mu \\
& +\sum_{n=2}^{\infty}\left[a_{n-1}\left(-b_{n-1}+b_{n}\right) \int_{\Delta} P_{n-1} d \mu \int_{\Delta} P_{n} d \mu\right. \\
& \left.+\left(-a_{n-1}^{2}+a_{n}^{2}\right)\left|\int_{\Delta} P_{n} d \mu\right|^{2}\right] \\
& \geq \mu(\Delta) \int_{\Delta}\left[a_{N}^{2}-\frac{1}{4}\left(\lambda-b_{N}\right)^{2}\right] P_{N}^{2}(\lambda) d \mu \\
& -\mu(\Delta) \int_{\Delta} P_{N}^{2} d \mu \sum_{2}^{N}\left[\left|a_{n-1}\left(-b_{n-1}+b_{n}\right)\right|+\left|-a_{n-1}^{2}+a_{n}^{2}\right|\right] \\
& -\mu(\Delta) \int_{\Delta} P_{N}^{2} d \mu \sum_{n=2}^{M}\left[\left|a_{n-1}\left(-b_{n-1}+b_{n}\right)\right|+\left|-a_{n-1}^{2}+a_{n}^{2}\right|\right] \\
& -\mid \sum_{M+1}^{\infty}\left[a_{n-1}\left(-b_{n-1}+b_{n}\right)\right] \int_{\Delta} P_{n-1} d \mu \int_{\Delta} P_{n} d \mu \\
& +\left(-a_{n-1}^{2}+a_{n}^{2}\right)\left(\int P_{n} d \mu\right)^{2} \\
& \geq \mu(\Delta) \frac{1}{32 k} \int_{\Delta} P_{N}^{2} d \mu-\frac{1}{32 k}|\mu(\Delta)|^{2} \geq \frac{1}{16 k}|\mu(\Delta)|^{2} \text {. }
\end{aligned}
$$

In each case, therefore, $\left\langle K E(\Delta) \phi_{1}, E(\Delta) \phi_{1}\right\rangle \geq(1 / 16 k)|\mu(\Delta)|^{2}$. It can also be shown (see the proof of Theorem 2.2.4 in [4]) that

$$
\left|\left\langle K E(\Delta) \phi_{1}, E(\Delta) \phi_{1}\right\rangle\right| \leq \frac{1}{2}\|J\||\Delta|\left\|E(\Delta) \phi_{1}\right\|^{2}
$$

where $|\Delta|$ denotes the length of $\Delta$. It therefore follows that $|\mu(\Delta)| \leq$ $8 k\|J\||\Delta|$. Let $\beta$ be a Borel subset of $(-1+1 / k, 1-1 / k)$ of Lebesgue measure zero. Then for any $\varepsilon>0$ there exists a pairwise disjoint sequence of intervals $\left\{\Delta_{j}\right\}$ such that $\Delta_{j} \subset(-1+1 / k, 1-1 / k), \beta \subset \cup \Delta_{j}$ and $\sum\left|\Delta_{j}\right|<\varepsilon$. Since $\mu(\beta) \leq \sum \mu\left(\Delta_{j}\right) \leq 8 k\|J\| \sum\left|\Delta_{j}\right|$ it follows that $\mu(\beta)=0$. 
Hence the spectral measure of $C_{k}$ is absolutely continuous on $(-1+$ $1 / k, 1-1 / k)$. By the Kato-Rosenblum Theorem the same result holds for the spectral measure of $C$.

CoROllaRY. Let $\lim a_{n}=\alpha(\alpha \neq 0)$ and $\lim b_{n}=b$. If

$$
\sum\left|-a_{n-1}^{2}+a_{n}^{2}\right|<\infty \text { and } \sum\left|a_{n-1}\left(-b_{n-1}+b_{n}\right)\right|<\infty
$$

then the spectral measure of $C$ is absolutely continuous on $(-2 \alpha+b$, $2 \alpha+b)$.

Finally it should be observed that the above theorem becomes false under the weaker condition that $K$ is compact. To see this assume that the main diagonal elements of $C$ are zero and define $a_{2 n-1}^{2}=1, a_{2 n}^{2}=$ $(n+1)^{2} / n^{2}$ for $n=1,2, \ldots$. Since $P_{n}^{2}(0)=\left(a_{n-2}^{2} / a_{n-1}^{2}\right) P_{n-2}^{2}(0)$ it follows that $P_{2 n}^{2}(0)=0$ and $P_{2 n+1}^{2}(0)=1 /(n+1)^{2}$. Therefore $\lambda=0$ is an eigenvalue. (See Stone [5].)

Note. A similar result has recently been attained by Mate and Nevai in [2] with very different techniques.

\section{REFERENCES}

[1] J. Dombrowski, Tridiagonal matrix representations of cyclic self-adjoint operators, to appear in Pacific Math.

[2] A Mate and P. Nevai, Orthogonal polynomials and absolutely continuous measures, preprint.

[3] P. Nevai, Orthogonal polynomials, Mem. Amer. Math. Soc., 18 (1979).

[4] C. R. Putnam, Commutation Properties of Hilbert space Operators and Related Topics, Ergebnisse der Math., 36, Springer, 1967.

[5] M. H. Stone, Linear transformations in Hilbert space, Amer. Math. Soc., New York, 1932.

Received January 20, 1984.

WRIGHT UNIVERSITY

DAYTON, OH 45435 
